\title{
Mean platelet volume in acute pancreatitis: a systematic review and meta-analysis
}

\author{
Vasileios P. Papadopoulos ${ }^{\mathrm{a}, \mathrm{b}}$, Dimitrios K. Filippou ${ }^{c}$, Konstantinos P. Mimidis ${ }^{\mathbf{b}}$ \\ ENARGEIA Medical Ltd., Xanthi; Democritus University of Thrace, Thrace, Alexandroupolis; National and \\ Kapodistrian University of Athens, Greece
}

Abstract

Background Several studies have suggested there may be statistically significant differences in mean platelet volume (MPV) between the onset and remission of acute pancreatitis (AP). This systematic review and meta-analysis aimed to better characterize the correlation between MPV and AP by identifying all relevant studies and summarizing their results.

Methods A comprehensive literature review was conducted using EMBASE, PubMed/MEDLINE, Cochrane Library, ClinicalTrials.gov, and Google Scholar from January 2000 to December 2019 to identify all studies that reported MPV at the onset or remission of AP, or both. Effect estimates from each study were extracted and combined using the random-effect, generic inverse variance method of DerSimonian and Laird. The Newcastle-Ottawa quality assessment scale was used to appraise the quality of the included studies.

Results Ten observational studies, including 1019 patients and 363 controls, were included in the meta-analysis. MPV was smaller at the onset of AP than on remission (standardized mean difference $=-0.33 \mathrm{fL}, 95 \%$ confidence interval -0.54 to $-0.12 \mathrm{fL} ; \mathrm{P}=0.002$ ); however, a moderate degree of heterogeneity $\left(I^{2}=72 \%, \mathrm{P} \leq 0.001\right)$ was observed. Subgroup analysis indicated comparable MPV in relation to the severity of AP. Similarly, no statistically significant difference was detected between $\mathrm{AP}$ patients and controls at either onset $(\mathrm{P}=0.760)$ or remission $(\mathrm{P}=0.700)$ of the disease. No statistically significant publication bias was detected (Eggers' regression $\mathrm{P}=0.938$ ). Subgroup analysis suggested age $(\mathrm{P}<0.001)$ and $\operatorname{sex}(\mathrm{P}=0.01)$ adjustment as potential sources of heterogeneity.

Conclusion MPV is smaller at the onset of AP. Further clinical evaluation is needed to assess its potential prognostic value.

Keywords Pancreatitis, mean platelet volume, blood platelets

Ann Gastroenterol 2020; 33 (4): 1-7

\section{Introduction}

It has been claimed that platelets are directly involved in the pathophysiology of acute pancreatitis (AP) [1]. An elevated

${ }^{a}$ Department of Internal Medicine, ENARGEIA Medical Ltd., Xanthi

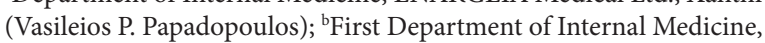
Democritus University of Thrace, Alexandroupolis (Vasileios P. Papadopoulos, Konstantinos P. Mimidis); 'Laboratory of Anatomy, Medical School, National and Kapodistrian University of Athens (Dimitrios K. Filippou), Greece.

Conflict of Interest: None

Correspondence to: Vasileios P. Papadopoulos, 6 Elpidos St., 67131

Xanthi, Greece, e-mail: vaspapmd@gmail.com

Received 28 January 2020; accepted 7 April 2020;

published online 15 May 2020

DOI: https://doi.org/10.20524/aog.2020.0495 activated platelet ratio has been observed at the onset of AP [2]. Moreover, greater platelet adhesiveness and aggregation has been documented in the early stages of the disease [3]. Mean platelet volume (MPV) has been proposed as a marker of platelet consumption and resulting compensatory bone marrow response during the disease process [2].

Several studies have reported statistically significant differences in MPV between the onset and remission of AP; furthermore, elevated MPV at admission has been linked with persistent organ failure in these patients [4]. However, the related literature remains obscure and thus needs further consolidation and clarification. Moreover, as properly designed prospective cohorts are still lacking, the potential prognostic value of MPV during the course of AP has so far remained elusive.

The present systematic review and meta-analysis was conducted with the aim of providing further evidence regarding a potential correlation between MPV and AP by identifying all relevant studies and summarizing their results. 


\section{Materials and methods}

A systematic literature review was conducted using EMBASE, PubMed/MEDLINE, Cochrane Library, and ClinicalTrials.gov databases from January 2000 to December 2019 to identify all studies that reported MPV at the onset or remission of AP, or both. The Google Scholar database was used as an additional pool of published data; an iterative search was performed until no additional publications could be traced. Lastly, we searched for unpublished dissertations and other unpublished work. The relevant protocol was submitted to the PROSPERO database (ID: 150901).

The review was independently conducted by 2 authors (VP and DF) using a search strategy that included the terms "mean platelet volume", "MPV" and "pancreatitis"; a third author (KM) was responsible for resolving any discordance. No software was used for study retrieval. Sources of financial support were traced where possible. The present study was conducted in accordance with the PRISMA (Preferred Reporting Items for Systematic reviews and Meta-Analyses) guidelines to formulate the basis of pre-specified eligibility criteria using the PICO (PPopulations/People/Patient/Problem: Patients in acute phase of AP and controls, I-Intervention(s): AP, C-Comparison: between patients at onset and remission (at discharge or at least 7 days after admission) of AP; between patients at onset of AP and controls; between patients at remission of AP and controls; between severe and mild cases at onset of AP, O-Outcome: MPV) worksheet and search strategy [5]. The AMSTAR checklist was used to confirm the high quality of the present meta-analysis [6].

Eligible studies were all that: 1) were written in English; 2) were case-control studies; 3) had a consistent outcome of interest; 4) reported a measure of statistical significance; 5) reported an effect size (means accompanied by their standard deviations); and 6) reported effect estimates not already reported.

The Newcastle-Ottawa quality assessment scale (NOS) was used to appraise the quality of the included studies in 3 areas: namely, the identification and recruitment of participants, the comparability between the 2 groups and the ascertainment of the exposure of interest [7]. Kappa statistics were used for the evaluation of inter-rater agreement in the case of NOS.

A structured data collection form was used to extract the following data from each study: title of the study, name of the first author, year of publication, country where the study was conducted, number of patients, severity of cases (if reported), number of controls (if any), correlation coefficient between paired data (if known), MPV of patients at onset of AP, MPV of patients at remission of AP, MPV of controls and adjustment for potential confounders (sex, age and body mass index). The data extraction process was carried out by 2 authors (VP and $\mathrm{DF})$; a third author (KM) was responsible for cross-checking in case of any discordance.

Data analysis was performed using Revman 5.3 software from the Cochrane Collaboration (London, United Kingdom). As effect estimates, standardized mean differences (SMD) and confidence intervals (CI) expressed in fL were utilized.
Effect estimates from each study were combined together using the random-effect, generic inverse variance method of DerSimonian and Laird, which assigned the weight of each study in the pooled analysis inversely to its variance [8]. In cases that median and interquartile range (IQR) were available, mean was considered equal to median and standard deviation (SD) was approached by the formula IQR/1.35 if normal distribution was reported; otherwise, the.xls tool based on the approach of Wan et al was applied [9].

As data between onset and remission of AP are paired, pooled $\mathrm{SD}$ was used, as approached by the formula $\left[\mathrm{SD}_{\text {onset }}{ }^{2}+\mathrm{SD}_{\text {remis }}^{2}\right.$ - $\left.2 \times \mathrm{r} \times \mathrm{SD}_{\text {onset }} \times \mathrm{SD}_{\text {remis }}\right]^{1 / 2}$, where $\mathrm{SD}_{\text {onset }}, \mathrm{SD}_{\text {remis }}$ and $\mathrm{r}$ denote $\mathrm{SD}$ at onset of $\mathrm{AP}, \mathrm{SD}$ at remission of $\mathrm{AP}$ and the correlation coefficient between data. In cases where $r$ was unknown, it was arbitrarily given a value of 0.5 if statistical significance was reported; otherwise, the $r$ value was nullified. Analysis of publication bias was performed through Eggers' regression, funnel plot with trim-and-fill analysis, standardized residual histogram, Galbraith plot, normal quintile plot, Rosenthal failsafe- $\mathrm{N}$ test, Orwin failsafe-N test and Gleser \& Olkin number of unpublished studies with the aid of Meta-Essentials software [10]. Analysis of heterogeneity, as derived from the $\mathrm{Q}$ test and $I^{2}$ statistic (Q test $\mathrm{P}$-value $<0.10$ was indicative of a statistically significant result; furthermore, a value of $I^{2} \leq 25 \%$ was indicative of insignificant heterogeneity, 26-50\% of low heterogeneity, $51-75 \%$ of moderate heterogeneity and $>75 \%$ of high heterogeneity), was performed through meta-regressions focusing on study characteristics, biases and confounders; multivariate analysis as well as subgroup analysis followed in case of univariate $\mathrm{P}<0.1$ [11]. All statistical tests were carried out using SPSS 20.0 software (IBM Corp C) $)$

\section{Results}

Forty-nine potentially relevant publications were identified through a thorough search of the literature; 15 were retrieved from EMBASE, 13 from PubMed/MEDLINE, 4 from ClinicalTrials.gov, and 17 from Google Scholar databases. No relevant publication was recognized in the Cochrane Library. No unpublished data of interest were detected.

After the exclusion of 31 duplicates, all the remaining 18 publications were initially reviewed based only on title and abstract; 5 failed to fulfill the eligibility criteria based on language, type of article, study design, and measured outcomes. The remaining 13 publications were reviewed based on full text; 3 were excluded from the meta-analysis ( 1 letter and 2 reporting irrelevant outcomes). Finally, 10 case-control studies with 1019 patients and 363 controls were included in the metaanalysis (Fig. 1).

All the characteristics of the studies and their quality assessment are presented in Table 1. The inter-rater agreement for NOS was high (kappa=0.78). The relevant data did not reveal any profound quality handicap.

The pooled analysis demonstrated that MPV was lower at the onset of AP than at remission of the disease ( $\mathrm{SMD}=-0.33 \mathrm{fL}, 95 \% \mathrm{CI}-0.54$ to $-0.12 \mathrm{fL}$; $\mathrm{P}=0.002$ ); however, 


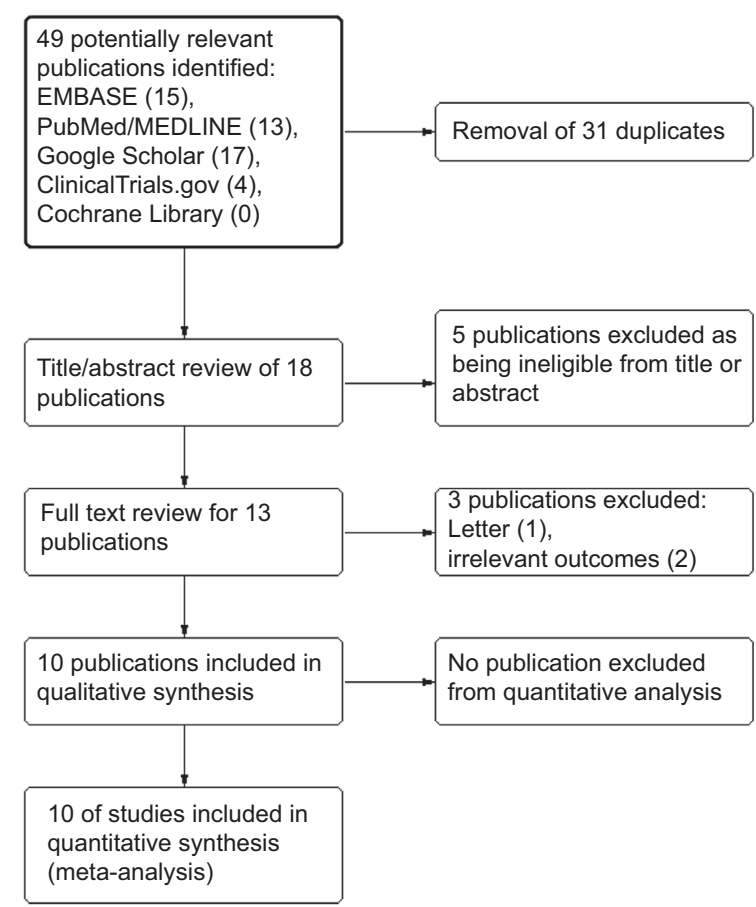

Figure 1 Flow chart

moderate heterogeneity $\left(I^{2}=72 \%, \mathrm{P}<0.001\right)$ was observed. Subgroup analysis indicated comparable MPV in relation to the severity of AP (SMD=-0.42 fL, 95\%CI -1.02 to 0.17 ; $\mathrm{P}=0.170)$. Similarly, no statistically significant difference was detected between AP patients and controls at either onset $(\mathrm{SMD}=0.12$ $\mathrm{fL}, 95 \% \mathrm{CI}-0.61$ to $0.84 \mathrm{fL} ; \mathrm{P}=0.760$ ) or remission ( $\mathrm{SMD}=0.07$ fL, $95 \% \mathrm{CI}-0.31$ to $0.46 \mathrm{fL} ; \mathrm{P}=0.700$ ) of the disease (Fig. 2). No statistically significant publication bias was detected (Eggers' regression $\mathrm{P}=0.938$ ). Furthermore, the Rosenthal failsafe- $\mathrm{N}$ test rejected the ad-hoc rule (Failsafe- $\mathrm{N}=206$ ), Orwin failsafe-N was null and Gleser \& Olkin number of unpublished studies was 13 , fairly close to the number of studies included in the present meta-analysis. Moreover, the funnel plot was not indicative of lack of symmetry and trim-and-fill analysis produced no imputed data points (Fig. 3). The Galbraith plot is depicted in Supplementary Fig. 1.

No statistically significant model analyzing study characteristics and potential confounders explained heterogeneity by meta-regression (Table 2 ). However, subgroup analysis suggested age $(\mathrm{P}<0.001)$ and $\operatorname{sex}(\mathrm{P}=0.010)$ adjustment as potential sources of heterogeneity (Table 3 ).

\section{Discussion}

The question of whether platelets are directly involved in the pathophysiology of AP has remained controversial since it was first proposed in 2 studies of ours: the former concluded that an elevated activated platelet ratio has been observed at the onset of AP [2] and the latter that platelet adhesiveness and aggregation are greater in the early stages of the disease [3]. Furthermore, our team proposed that MPV could reflect platelet consumption and resulting compensatory bone marrow response during AP, thus being a potential candidate marker for the disease process, whose clinical usefulness remains to be tested [2]. Since then, several studies have been carried out in this area.

Three studies are in keeping with our results, having reported statistically significant differences in MPV between onset and remission of AP $[13,18,19]$. In 2 of them, the result was independent of the severity of the disease $[18,19]$. Interestingly, Beyazit et al [13] discriminated between mild and severe AP, concluding that MPV has a crucial value in determining disease severity; furthermore, comparisons of MPV with other inflammatory markers, such as C-reactive protein (CRP), erythrocyte sedimentation rate (ESR), and white blood cell count (WBC) reported better overall accuracy for the former $(72.7 \%$ at a cutoff of $7.85 \mathrm{fL})$. In keeping with the above mentioned results, Erbis et al proposed a very similar cutoff (7.80 fL) to discriminate between necrotizing and nonnecrotizing AP [17]. Additionally, a cutoff of $12 \mathrm{fL}$ was used as a prognostic indicator of persistent organ failure in patients with AP in the work carried out by Huang et al [4].

In contrast, Osada et al reported comparable MPV values between the onset and remission of AP [14]. This study underlines that platelets, which provide the cellular link between the inflammatory response and the activation of coagulation, may play an important role in the initiation of AP and the development of serious complications. The authors evaluated platelet morphology along with functional parameters in relation to the severity of AP, and concluded that patients with severe AP presented significantly lower MPV than controls at disease onset. Furthermore, they stated that patients with AP, independently of disease severity, exhibited an increase in high volume platelets at remission of the disease and proposed that this phenomenon could be explained in terms of reactive thrombocythemia. In keeping with the latter, Kefeli et al reported a significantly increased platelet count at remission of AP in comparison with the onset of disease; however, the authors reported comparable MPV at onset and remission of AP, as well as in controls [16]. Yarkaç et al also reported comparable MPV values between the onset and remission of AP and their best cutoff value for MPV to discriminate mild from severe cases of AP (9.4 fL) failed to reach statistical significance $(\mathrm{P}=0.067)$ [20].

Akbal et al reported a greater MPV at the onset of AP when compared with controls; however, they found comparable MPV values between the onset and remission of AP. Furthermore, the authors reported that MCV was correlated with D-dimers and fibrinogen levels but not with inflammation markers (CRP, ESR, and WBC) proposing that higher MPV levels in acute pancreatitis may reflect hypercoagulation associated with the disease [15].

As far as the etiology of AP is concerned, Okuturlar et al reported lower MPV in patients with non-biliary AP compared with biliary AP at both onset and remission of the disease, attributing the observed difference to early-onset infection in non-biliary AP patients [18]. However, Yarkaç et al failed to reproduce this finding [20]. 


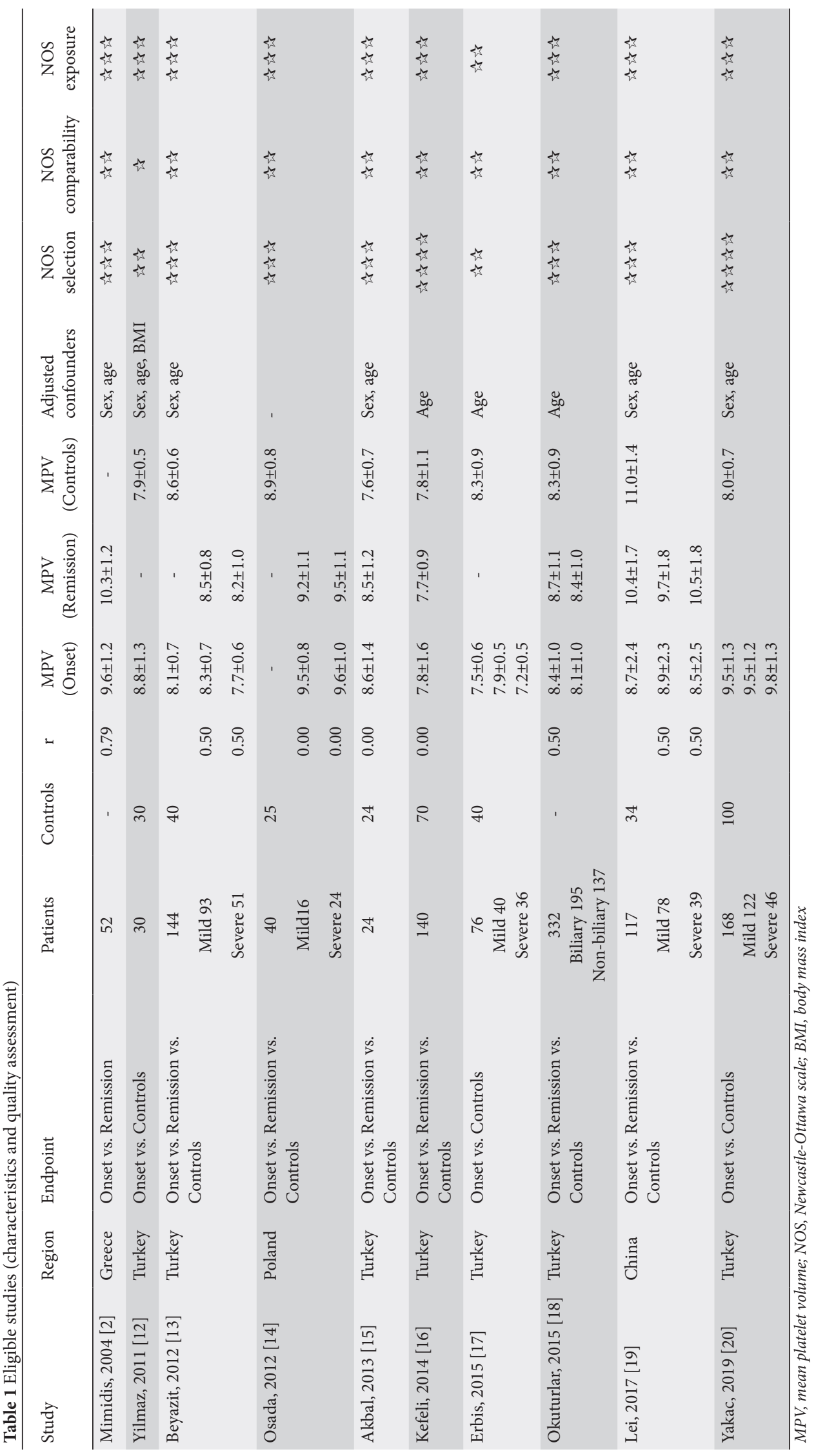




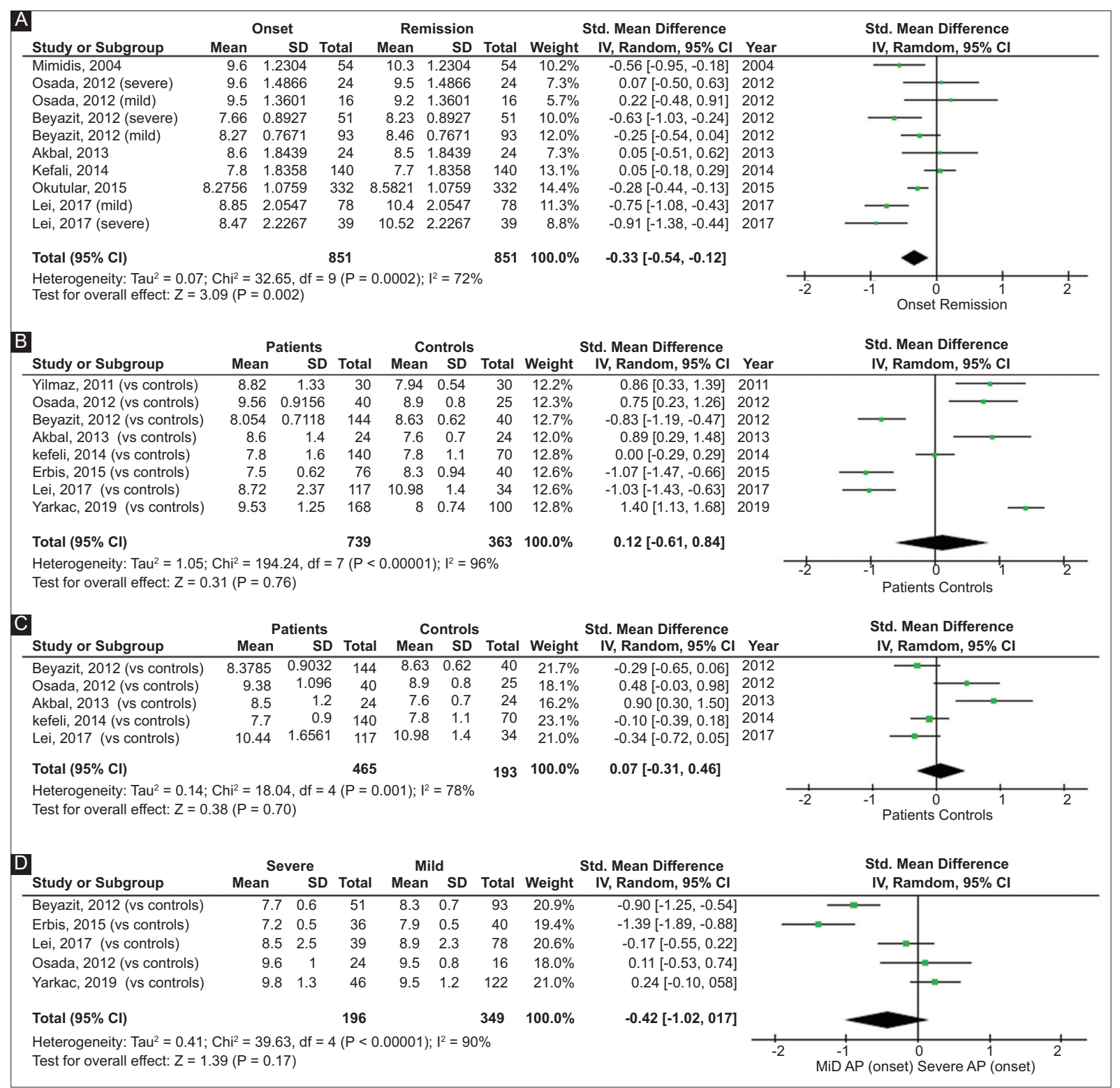

Figure 2 Meta-analysis (A) Onset vs. Remission, (B) Onset vs. Controls, (C) Remission vs. Controls and (D) Severe vs. Mild cases of AP at onset

Table 2 Meta-regression analysis

\begin{tabular}{lcccc}
\hline Parameter & Univariate analysis $(\mathrm{r})$ & $\begin{array}{c}\text { Univariate } \\
\text { P-value }\end{array}$ & $\begin{array}{c}\text { Meta-regression analysis } \\
\text { (standardized beta) }\end{array}$ & $\begin{array}{c}\text { Meta-regression } \\
\text { P-value }\end{array}$ \\
\hline Study characteristics & & & \\
$\quad$ Sample size & -0.014 & 0.969 & \\
Years passed from publication & 0.162 & 0.655 & & \\
Cohort study & -0.161 & 0.656 & -0.277 & 0.336 \\
Including severe cases & -0.068 & 0.852 & -0.304 & 0.322 \\
Adjustment for confounders & & & \\
Age & -0.592 & 0.036 & 0.015 & \\
Sex & -0.684 & & & \\
\hline
\end{tabular}




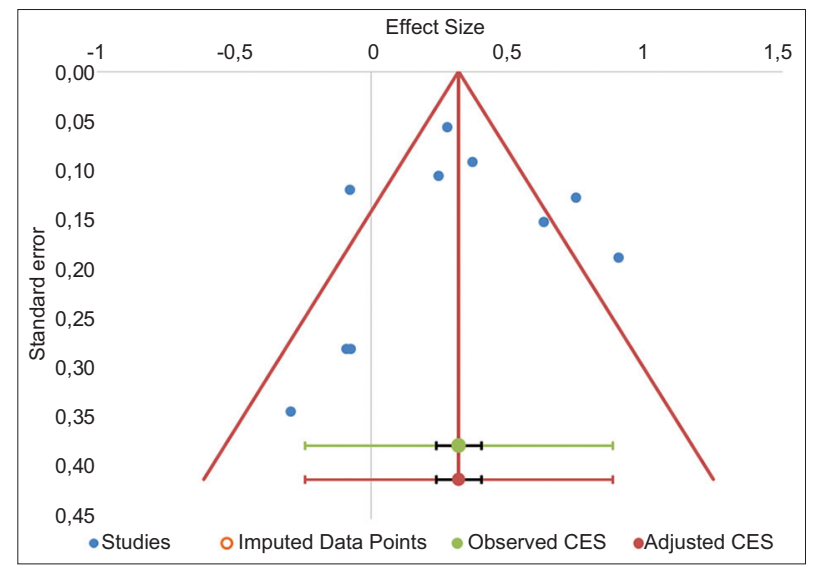

Figure 3 Funnel plot with trim-and-fill analysis CES, combined effect size

Table 3 Subgroup analysis

\begin{tabular}{llcccc}
\hline Parameter & Subgroups & SMD & $95 \% \mathrm{CI}$ & $I^{2}$ & P-value \\
\hline Age adjustment & Yes & -0.21 & -0.40 to -0.03 & $58 \%$ & $<0.001$ \\
& No & -0.80 & -1.07 to -0.54 & $0 \%$ & \\
Sex adjustment & Yes & -0.52 & -0.77 to -0.27 & $60 \%$ & 0.01 \\
& No & -0.07 & -0.32 to 0.19 & $60 \%$ & \\
\hline
\end{tabular}

SMD, standardized mean difference; CI, confidence interval

Lei at al studied the time course of MPV alterations in AP by measuring it at admission (day 1), and on days 2, 3 and 7 . The authors reported a lower MPV in AP patients at admission compared to days 2, 3 and 7, while they suggested that an MPV less than $6.65 \mathrm{fL}$ at admission for AP could predict organ failure [19]. However, whether MPV could possibly serve as a predictor of the severity of AP as well as of complications of the disease needs further, carefully designed clinical studies.

Our meta-analysis incorporated all the above mentioned data, simultaneously performing quality assessment, publication bias analysis, subgroup analyses and metaregressions. Although NOS cannot discriminate between studies of "high" and "low" quality, it constitutes a valuable tool for forming inclusion criteria for the meta-analysis, informing a sensitivity analysis or meta-regression, weighting studies, or highlighting areas of methodological quality poorly addressed by the included studies [21]. Subgroup analyses and metaregressions focused on the potential effect of study type, sample size, region of origin, confounders, and combined outcomes.

Interestingly, no publication bias was detected, as implied by results derived from Eggers' regression, funnel plot with trimand-fill analysis, standardized residual histogram, Galbraith plot, normal quintile plot, Rosenthal failsafe-N test, Orwin failsafe-N test and Gleser \& Olkin number of unpublished studies; this result could be attributed to the fact that no clearcut predefined or prejudged size or even direction of difference was suspected in the scientific community as a whole.

No statistically significant model analyzing study characteristics and confounders explained the observed increased heterogeneity $\left(I^{2}=72 \%, \mathrm{P} \leq 0.001\right)$ by meta-regression. However, subgroup analysis revealed age $(\mathrm{P}<0.001)$ and sex $(\mathrm{P}=0.010)$ adjustment as potential sources of heterogeneity. These findings are in keeping with the current knowledge and literature, as age and sex are known determinants for AP; the incidence of AP increases with age and it is twice more common in males than females worldwide [22]. All the above underlie the need for careful interpretation of data already published, as well as study design and performance in the future.

Summing up, the present meta-analysis suggested that MPV is higher at remission of AP than at the onset of the disease, and subgroup analysis indicated comparable MPV in relation to AP severity. However, no statistically significant difference was detected between AP patients and controls at either onset or remission of the disease. These findings are in keeping with initial platelet activation and wear, resulting in lower MPV, and subsequent reactive thrombocythemia leading to an increase of large platelets during inflammation [23].

The inconsistencies that the above-mentioned findings may seem to convey could at first be attributed to the fact that the data were derived from different studies for each comparison, as well as to the substantial heterogeneity. Seemingly, mean MPV values, despite being lower at the onset of AP than at remission of the disease, could be supposed to lie within the normal range in both cases.

The major limitation of the present study might be that the data analyzed were derived from case-control studies. Interestingly though, meta-regression did not prove any statistically significant difference regarding overall effect sizes; however, due to the fact the pooled studies were observational, residual confounding is a major limitation and even the meta-regression cannot control unknown confounders. Thus, this practice might be considered nondecisive. Furthermore, the present study failed to incorporate unpublished data; lack of this kind of source might be linked to potential publication bias, despite the fact that no prejudiced correlation between MPV and AP (positive or negative) prevailed in the literature. Such biases might not be traced in small-sized studies including less than 10 studies due to the fact that funnel plots, as well as any statistical tool used for the same purpose, are underpowered.

In conclusion, our results suggest that MPV is lower at the onset of AP than at remission of the disease, independently of the disease severity. As MPV constitutes an inexpensive and undemanding marker, it would be reasonable to further investigate its potential prognostic value. Therefore, further specially designed clinical evaluations would be needed to assess the clinical usefulness of MPV in everyday clinical practice.

\section{References}

1. Kakafika A, Papadopoulos V,Mimidis K, MikhailidisDP.Coagulation, platelets, and acute pancreatitis. Pancreas 2007;34:15-20.

2. Mimidis K, Papadopoulos V, Kotsianidis J, et al. Alterations of 


\section{Summary Box}

\section{What is already known:}

- A higher activated platelet ratio has been observed at the onset of acute pancreatitis (AP)

- Greater platelet adhesiveness and aggregation has been documented in the early stages of AP

- Studies have reported statistically significant differences in mean platelet volume (MPV) between the onset and remission of AP

\section{What the new findings are:}

- MPV is smaller at the onset of AP

- MPV is independent of the severity of AP

- MPV, an inexpensive and undemanding marker, deserve further investigation of its potential prognostic value in AP

platelet function, number and indexes during acute pancreatitis. Pancreatology 2004;4:22-27.

3. Mimidis K, Papadopoulos V, Kartasis Z, et al. Assessment of platelet adhesiveness and aggregation in mild acute pancreatitis using the PFA-100 system. JOP 2004;5:132-137.

4. Huang P, Zhang Y, Wu H. Mean platelet volume as an indicator of persistent organ failure in acute pancreatitis. Int J Clin Exp Pathol 2016;9:12883-12889.

5. Moher D, Shamseer L, Clarke M, et al; PRISMA-P Group. Preferred reporting items for systematic review and meta-analysis protocols (PRISMA-P) 2015 statement. Syst Rev 2015;4:1.

6. Shea BJ, Reeves BC, Wells G et al. AMSTAR 2: a critical appraisal tool for systematic reviews that include randomised or nonrandomised studies of healthcare interventions, or both. $B M J$ 2017;358:j4008.

7. Stang A. Critical evaluation of the Newcastle-Ottawa scale for the assessment of the quality of nonrandomized studies in metaanalyses. Eur J Epidemiol 2010;25:603-605.

8. DerSimonian R, Laird N. Meta-analysis in clinical trials. Control
Clin Trials 1986;7:177-188.

9. Wan X, Wang W, Liu J, Tong T. Estimating the sample mean and standard deviation from the sample size, median, range and/or interquartile range. BMC Med Res Methodol 2014;14:135.

10. Suurmond R, van Rhee H, Hak T. Introduction, comparison, and validation of meta-essentials: a free and simple tool for metaanalysis. Res Synth Methods 2017;8:537-553.

11. Higgins JP, Thompson SG, Deeks JJ, Altman DG. Measuring inconsistency in meta-analyses. BMJ 2003;327:557-560.

12. Yilmaz N, Ozkan OV, Buyukbas S, et al. Mean platelet volume in patients with acute pancreatitis. J Clin Exp Invest 2011;2:362-365.

13. Beyazit Y, Sayilir A, Torun S, et al. Mean platelet volume as an indicator of disease severity in patients with acute pancreatitis. Clin Res Hepatol Gastroenterol 2012;36:162-168.

14. Osada J, Wereszczynska-Siemiatkowska U, Dabrowski A, Dabrowska MI. Platelet activation in acute pancreatitis. Pancreas 2012;41:1319-1324.

15. Akbal E, Demirci S, Koçak E, Köklü S, Başar O, Tuna Y. Alterations of platelet function and coagulation parameters during acute pancreatitis. Blood Coagul Fibrinolysis 2013;24:243-246.

16. Kefeli A, Basyigit S, Özgür Yeniova A, Küçükazman M, Nazligül Y, Aktas B. Platelet number and indexes during acute pancreatitis. Euroasian J Hepatogastroenterol 2014;4:67-69.

17. Erbis H, Aliosmanoglu I, Turkoglu MA, Ay E, Turkoglu A, Ulger BV. Evaluating mean platelet volume as a new indicator for confirming the diagnosis of necrotizing pancreatitis. Ann Ital Chir 2015;86:132-136.

18. Okuturlar Y, Soylu A, Dogan H, et al. Mean platelet volume in patients with biliary and non-biliary acute pancreatitis. Int J Clin Exp Pathol 2015;8:2051-2056.

19. Lei JJ, Zhou L, Liu Q, Xiong C, Xu CF. Can mean platelet volume play a role in evaluating the severity of acute pancreatitis? World J Gastroenterol 2017;23:2404-2413.

20. Yarkaç A, Köse A, Bozkurt Babuş S, Ateş F, Örekici Temel G, Ölmez A. The value of hematological parameters in acute pancreatitis. Ulus Travma Acil Cerrahi Derg 2019;25:453-460.

21. Stang A. Critical evaluation of the Newcastle-Ottawa scale for the assessment of the quality of nonrandomized studies in metaanalyses. Eur J Epidemiol 2010;25:603-605.

22. Petrov MS, Yadav D. Global epidemiology and holistic prevention of pancreatitis. Nat Rev Gastroenterol Hepatol 2019;16:175-184.

23. Korniluk A, Koper-Lenkiewicz OM, Kamińska J, Kemona H, Dymicka-Piekarska V. Mean platelet volume (MPV): new perspectives for an old marker in the course and prognosis of inflammatory conditions. Mediators Inflamm 2019;2019:9213074. 


\section{Supplementary Figure}

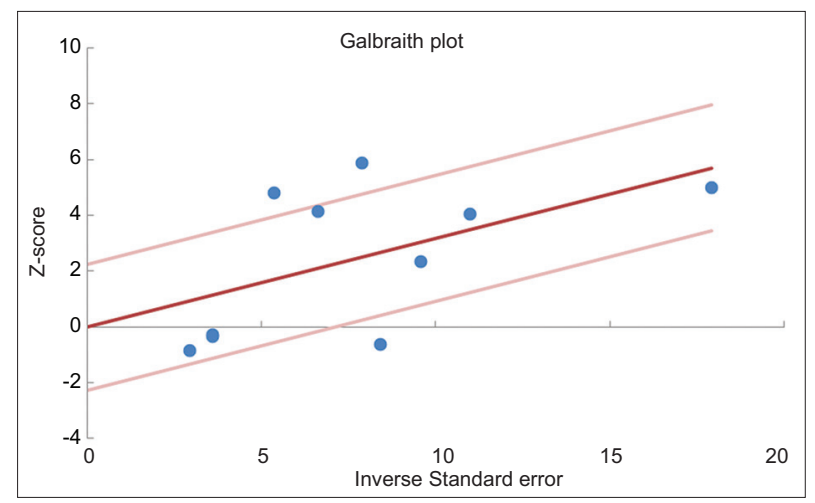

Supplementary Figure 1 Galbraith plot 